\title{
In Situ TEM Observation on Formation of Uniform Amorphous Layer on $\mathrm{SnO}_{2}$ Nanotube
}

\author{
Joon Ha Chang ${ }^{1,2}$, Jun Young Cheong ${ }^{2}$, Il-Doo Kim² and Jeong Yong Lee ${ }^{1,2}$ \\ 1. Center for Nanomaterials and Chemical Reactions, Institute for Basic Science (IBS), Daejeon, \\ Korea (305-701) \\ 2. Department of Materials Science and Engineering, Korea Advanced Institute of Science and \\ Technology, Daejeon, Korea (305-701)
}

Interfacial reactions at electrolyte/electrode boundary are crucially important for the performance of lithium-ion batteries (LIBs) as they are one of influencing parameters in the transport of electrons and $\mathrm{Li}$ ions to the electrodes [1,2]. Taking into account that these interfacial reactions are important, a number of studies utilizing Fourier Transform Infrared spectroscopy (FTIR) analysis and x-ray photoelectron spectroscopy (XPS) have been reported in this subject [3,4]. However, limitations exist as there they are based on ex situ analysis, where they were examined in the electrode that was stripped off from the electrochemical cell and underwent washing process, where some variables exist [5]. To overcome these limitations, in situ analysis was adopted beyond the simple research on the morphology and composition of the final products, in order to fully understand the mechanisms occurring at interfacial layers.

As for the electrochemical cells or energy storage system, different kinds of layers are formed upon subsequent electrochemical reactions. For the lithium-ion batteries (LIBs), solid electrolyte interphase (SEI) layer plays an important role as it determines different parameters of battery, from cyclability to c-rate capability. Surprisingly, lack of in situ observation of such formation process of SEI layer is yet exactly known. The exact formation process of SEI layer upon lithiation still remains unknown, although it is an important phenomenon to observe. Especially, whether the SEI layer is formed on the surface of the active material with non-uniform thickness or uniform thickness remains unsolved challenge that needs to be addressed, to possibly come up with more smart material designs that may modulate the interfacial properties of the given material.

Experiment was conducted by following steps: 1) synthesis of monolayer graphene on $\mathrm{Cu}$ foil by chemical vapor depositon (CVD) process and 2) trapping electrolyte containing $\mathrm{LiPF}_{6}$ and $\mathrm{SnO}_{2}$ nanotubes between the two graphene sheets. Figure 1 shows the HRTEM image of $\mathrm{SnO}_{2}$ nanotube, indicating (110) plane of $\mathrm{SnO}_{2}$ nanotube. Real-time observation of uniform amorphous layer formation is shown in Figure 2. At initial state $(0 \mathrm{~s})$, very thin layer $(\approx 2 \mathrm{~nm})$ was as-formed. As time passed to $30 \mathrm{~s}$, the thickness of the amorphous layer became $3 \mathrm{~nm}$ and $4.2 \mathrm{~nm}$ at $130 \mathrm{~s}$. Finally, the amorphous layer grew up to $5 \mathrm{~nm}$ and no further change in thickness occurred. We believe the termination of reaction is due to limited amount of $\mathrm{Li}$ and electrolyte since very small amount of liquid is encapsulated around the nanotube. It can be suggested that sub-nanoscale sources of decomposed electrolytes are deposited uniformly on both the surface of $\mathrm{SnO}_{2}$ nanotube, with uniform thickness and morphology.

In summary, using GLC-TEM, direct observation of growth of amorphous layer was shown in $\mathrm{SnO}_{2}$ nanotube in liquid electrolyte system. Formation of uniform and stable layer was found, which is important in cyclability and coulombic efficiency in real LIB. Further examination of formation of such uniform interfacial layers will contribute in designing both high-performance and stable LIBs.[6] 


\section{References:}

[1] Wu, H. et al, Nature Nanotechnol. 7 (2012), p. 310-315.

[2] D. Aurbach et al, J. Power Sources 162 (2006), p.780-789.

[3] Y. Ein-Eli et al, Electrochim. Acta 39 (1994), p. 2559.

[4] D. Aurbach et al, Langmuir 12 (1996), p. 3991.

[5] P. Verma, P. Maire and P. Novak, Eectrochim. Acta 55 (2010), p. 6332-6341.

[6] The authors acknowledge the funding from Korea CCS R\&D Center (KCRC) grant by the Korea government (Ministry of Science, ICT \& Future Planning) (No. NRF-2014M1A8A1049303), EndRun grant from KAIST by the Korea government in 2015 (Ministry of Science, ICT \& Future Planning) (N01150615), and Institute for Basic Science (IBS) (IBS-R004-G3).

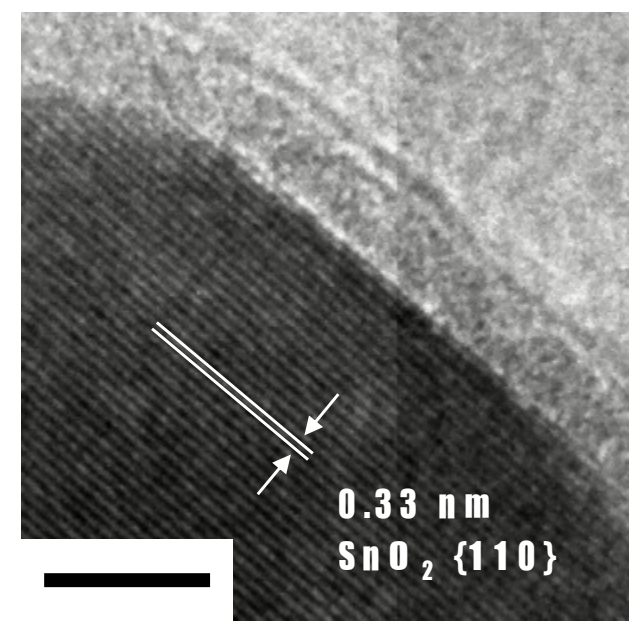

Figure 1. HRTEM image showing (110) plane of $\mathrm{SnO}_{2}$ nanotube. (Scale bar $=5 \mathrm{~nm}$ )
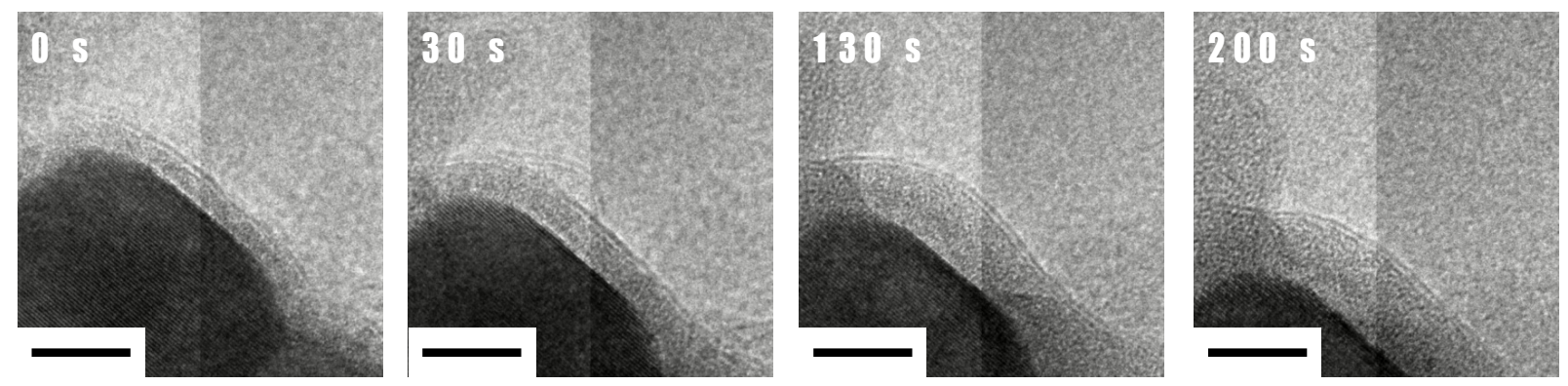

Figure 2. Time-series TEM snapshots of movie showing the formation of uniform amorphous layer on $\mathrm{SnO}_{2}$ nanotube. $($ Scale bar $=10 \mathrm{~nm})$ 